\title{
EL NÚMERO ÓPTIMO DE EMPRESAS BAJO COMPETENCIA DE BERTRAND
}

\author{
THE OPTIMAL NUMBER OF FIRMS UNDER BERTRAND \\ COMPETITION
}

Germán Coloma*

\begin{abstract}
Resumen
Este trabajo es acerca de un modelo de competencia en precios en el mercado de un producto homogéneo con libre entrada de empresas idénticas y rendimientos variables a escala. Si el número óptimo de empresas activas en el mercado es dos o más, y el número de empresas activas es igual a dicho número óptimo, entonces el equilibrio de Bertrand existe para dicho número óptimo, y no existe si el número de empresas activas es menor que el óptimo. El modelo, sin embargo, no descarta la existencia de equilibrios con más empresas activas que el óptimo. Finalmente, si el número óptimo de empresas es igual a uno, entonces el equilibrio de Bertrand no existe.
\end{abstract}

Palabras clave: Equilibrio de Bertrand, Rendimientos variables, Libre entrada, Número de empresas.

\begin{abstract}
This paper is about a model of Bertrand competition in a homogeneous-good market with free entry of identical firms and variable returns to scale. If the optimum number of active firms in the market is two or more, and the number of active firms is equal to that optimum number, then Bertrand equilibrium exists for that optimum number, and it does not exist if the number of active firms is less than the optimum. The model, however, does not rule out the existence of Bertrand equilibria with more active firms than the optimum number. Finally, when the optimum number of active firms in the market is one, Bertrand equilibrium does not exist.
\end{abstract}

Key words: Bertrand equilibrium, Variable returns to scale, Free entry, Number of firms.

JEL Classification: D43, L13.

* Universidad del CEMA; Av. Córdoba 374, Buenos Aires, C1054AAP, Argentina. Teléfono: 54-11-6314-3000. Correo electrónico: gcoloma@cema.edu.ar. Agradezco los comentarios de Leandro Arozamena, Rómulo Chumacero, Giacomo Corneo, Simon Grant, Jorge Streb, Federico Weinschelbaum, Makoto Yano y dos árbitros anónimos. 


\section{INTRODUCCIón}

Desde que fue tratado por primera vez por Marshall (1890), el tema del número óptimo de empresas (y su comparación con el número de empresas de equilibrio en un mercado) se volvió tradicional en el análisis económico de la competencia perfecta. Posteriormente recibió también atención para casos de competencia de Cournot (Mankiw y Whinston, 1986; Corchon y Fradera, 2002), competencia monopolística (Dixit y Stiglitz, 1977; Anderson et al., 1995) y colusión (Brander y Spencer, 1985; Friedman y Thisse, 1994).

En este trabajo consideraremos la relación entre el número óptimo de empresas y el número de empresas de equilibrio bajo competencia de Bertrand, en mercados de productos homogéneos con libre entrada. Para ello desarrollaremos un modelo con empresas idénticas que poseen una clase particular de función de costos con rendimientos variables a escala, consistente en la suma de un costo variable convexo y de un costo fijo evitable. Empresas idénticas y rendimientos variables son requisitos virtualmente indispensables para analizar el número óptimo de empresas en un mercado. Si las empresas no son idénticas, el problema implica también definir cuáles empresas deberían estar activas y cuáles no, y no simplemente la determinación de un número óptimo de empresas activas. Por el contrario, si los rendimientos a escala no son variables, encontrar el número óptimo de empresas se vuelve trivial: es igual a uno si los rendimientos son crecientes, tiende a infinito si son decrecientes, y es irrelevante si los rendimientos a escala son constantes.

Un punto característico que suelen tratar los trabajos sobre comparación entre número óptimo de empresas y número de empresas en equilibrio es la posible coincidencia o divergencia entre ambos conceptos, siendo el resultado clásico al respecto la idea de que, mientras los dos conceptos coinciden en mercados de competencia perfecta, los mismos divergen en otros tipos de mercados. En este trabajo veremos que, para el caso de la competencia de Bertrand con productos homogéneos y rendimientos variables a escala, el número de empresas en equilibrio puede coincidir con el número óptimo de empresas, pero que también puede divergir de un modo en particular. Dicho modo tiene que ver con el hecho de que, en nuestro modelo, el número de empresas en equilibrio puede llegar a ser mayor que el número óptimo, pero nunca menor.

Como los modelos de competencia en precios han sido desarrollados típicamente en contextos de rendimientos a escala no variables, la optimalidad del número de empresas en equilibrio bajo competencia de Bertrand en mercados con productos homogéneos es un tema bastante inusual en la literatura. Pero la libre entrada bajo competencia en precios sí ha sido analizada para diferentes situaciones de rendimientos crecientes como un modo de limitar el poder monopólico (Sharkey y Sibley, 1993; Chowdhury, 2002). También hemos hallado un trabajo que estudia la posible convergencia del equilibrio de Bertrand a un equilibrio de competencia perfecta en una situación de libre entrada con rendimientos variables (Novshek y Chowdhury, 2003), otro que trata sobre el tema de la libre entrada con rendimientos variables a escala en el contexto de una competencia del tipo Bertrand-Edgeworth (Yano, 2006), y otro que busca encontrar condiciones de largo plazo bajo las cuales cierto número de empresas, aunque no necesariamente el óptimo, se encuentra activo en un mercado (Ferreira y Dufourt, 2007). 
El presente trabajo también puede incluirse dentro de la literatura sobre la relación entre el equilibrio de Bertrand y la "desafiabilidad" de los mercados (market contestability), de la cual son ejemplos Chaudhuri (1998) y D’Aspremont, Ferreira y Gérard-Varet (2000), o verse como una continuación de un trabajo previo sobre el equilibrio de Bertrand en mercados con rendimientos variables a escala (Saporiti y Coloma, 2010). Ese último artículo es en rigor una extensión de la literatura sobre equilibrio de Bertrand cuando las empresas tienen funciones de costos variables convexas (Dastidar, 1995; Weibull, 2006) a un caso en el cual las mismas también afrontan un costo fijo evitable o "no hundido"1.

Este trabajo, sin embargo, tiene una diferencia fundamental con Saporiti y Coloma (2010), ya que en aquel trabajo no se analiza la posible entrada de empresas al mercado ni se estudia el tema del número óptimo de empresas activas. De hecho, la principal contribución de Saporiti y Coloma (2010) tiene que ver con un resultado de existencia del equilibrio de Bertrand en casos de funciones de costos superaditivas, y de inexistencia del mismo en casos de funciones de costos subaditivas. Dicho resultado también aparecerá tangencialmente en el presente trabajo, cuando analicemos situaciones en las cuales el número óptimo de empresas en el mercado es igual a uno.

\section{DESCRIPCIÓN DEL MODELO}

Consideremos el mercado de un producto homogéneo con muchas empresas potencialmente oferentes. Cada una de ellas tiene la siguiente función de costos con rendimientos variables a escala:

$$
C\left(Q_{i}\right)=\left\{\begin{array}{ll}
0 & (\text { si } \mathrm{Qi}=0) \\
V C\left(Q_{i}\right)+F & (\text { si } \mathrm{Qi}>0)
\end{array} ;\right.
$$

donde $Q_{i}$ es la cantidad ofrecida por la iésima empresa, $V C$ en una función continua, creciente y estrictamente convexa de $Q_{i}$, y $F$ es un parámetro no negativo que representa un costo fijo evitable o no hundido. Esta función de costos puede expresarse también como una función de costo medio $A C\left(Q_{i}\right)=C\left(Q_{i}\right) / Q_{i}$.

Supongamos que en este mercado hay $n$ empresas activas (es decir, $n$ empresas que ofrecen $Q_{i}>0$ ), y que las restantes empresas están inactivas (esto es, ofrecen $\left.Q_{i}=0\right)$. Respecto de cierta cantidad total $Q$, el número óptimo de empresas $(n *)$ es el número de empresas activas para el cual se da que $A C\left(Q / n^{*}\right) \leq A C(Q / m)$, para todo $m \neq n$ *.

Representemos la demanda agregada del mercado a través de la expresión $Q=D(P)$, donde $P$ es el precio pagado por los consumidores. Supongamos que $D$ es una función continua y decreciente de $P$, con $\lim _{P \rightarrow \infty} D(P)=0$ y $D(0)=K>0$. En ese caso podemos definir al número óptimo de empresas en el mercado

En Saporiti y Coloma (2010) se analiza también el caso intermedio en el cual parte de los costos fijos son inevitables o hundidos y otra parte son evitables o no hundidos. Dicho caso intermedio no juega ningún papel en el modelo presentado en el presente trabajo, en el cual supondremos que todos los costos fijos son evitables. 
como el número de empresas activas para el cual se da que $P_{L}\left(n^{*}\right) \leq P_{L}(m)$, para todo $m \neq n^{*}$, donde $P_{L}(n)=\min \left\{P: A C\left(D(P) / n^{*}\right)=P\right\}$ y $P_{L}(m)=\min \{P$ : $A C(D(P) / m)=P\} . P_{L}(n)$ es por lo tanto el "precio de autofinanciamiento" (breakeven price) para un caso con $n$ empresas activas en el mercado.

Supongamos ahora que, si este mercado está en una situación de competencia en precios, cada empresa enfrenta la siguiente función de demanda individual:

(2) $D_{i}\left(P_{i}, \mathbf{P}_{-\mathbf{i}}\right)=\left\{\begin{array}{cl}0 & (\text { si } \mathrm{Pi}>\mathrm{Pj}, \text { para algún } \mathrm{j} \neq \mathrm{i}) \\ \frac{\mathrm{D}\left(\mathrm{P}_{\mathrm{i}}\right)}{\mathrm{n}} & (\text { si } \mathrm{Pi}=\mathrm{Pj}, \text { para todo } \mathrm{j} \in \mathrm{N}, \mathrm{y} \mathrm{Pi}<\mathrm{Pk} \text {, para todo } \mathrm{k} \notin \mathrm{N}) \\ \mathrm{D}\left(\mathrm{P}_{\mathrm{i}}\right) & (\text { si } \mathrm{Pi}<\mathrm{Pj}, \text { para todo } \mathrm{j} \neq \mathrm{i})\end{array}\right.$

donde $P_{i}$ es el precio elegido por la iésima empresa, $P_{j}$ es el precio elegido por la jotaésima empresa, $P_{k}$ es el precio elegido por la k-ésima empresa, $n$ es el número de empresas activas, y $N$ es el conjunto de empresas activas.

Nótese que esta definición de la función de demanda de la empresa individual supone una "regla de reparto igualitario" (equal sharing rule), es decir, la idea de que si $n$ empresas cobran el mismo precio y ese precio es el mínimo precio cobrado por todo el conjunto de empresas, entonces la demanda se reparte equitativamente entre las $n$ empresas activas ${ }^{2}$. También implica que cada empresa activa debe satisfacer toda la demanda que enfrenta, igual a $D\left(P_{i}\right) / n$.

Nótese que, debido a este último requerimiento, el precio de autofinanciamiento $P_{L}(n)$ representa el mínimo precio al cual cada empresa activa puede obtener un beneficio no negativo. Dada la forma de la función de costos de las empresas individuales, sin embargo, existe también otro precio que genera un beneficio nulo, que es $P_{H}(n)=\max \{P: A C(D(P) / n)=P\}$, y que es el máximo precio al cual $n$ empresas activas pueden obtener de manera simultánea un beneficio no negativo. Supongamos además que, para todo $P \in\left(P_{L}(n) ; P_{H}(n)\right)$, se da que $P>A C(D(P) / n)$ y que, por lo menos para $n=1$, el intervalo $\left[P_{L}(n)\right.$; $\left.P_{H}(n)\right]$ no es un conjunto vacío.

La definición de demanda usada en este trabajo implica que cada empresa individual obtiene un beneficio $\prod_{i}$ igual a:

$$
\Pi_{i}=P_{i} \cdot D_{i}\left(P_{i}, P_{-i}\right)-C\left(D_{i}\left(P_{i}, P_{-i}\right)\right)
$$

lo cual quiere decir que cada empresa activa obtiene un beneficio igual a:

$$
\Pi_{i}=P_{i} \cdot \frac{D\left(P_{i}\right)}{n}-V C\left(\frac{D\left(P_{i}\right)}{n}\right)-F ;
$$

mientras que cada empresa inactiva obtiene un beneficio igual a cero.

2 Para ver otras reglas aplicables a situaciones de competencia en precios, véase Hoernig (2007). 
En una situación de competencia en precios como la descrita, un equilibrio de Bertrand es un vector de precios $\boldsymbol{P n}$ tal que, para todo $i \in N$ y todo $P_{\tilde{l}} \in$ $[0, \infty)$, se da que $\prod_{i}\left(P_{i}, \boldsymbol{P}_{-i}\right) \geq \prod_{i}\left(P_{\tilde{\imath}}, \boldsymbol{P}_{-i}\right)$ y, para todo $k \notin N$ y todo $P_{\tilde{\imath}} \in$ $[0, \infty)$, se da que $\prod_{k}\left(P_{\tilde{\imath}}, \boldsymbol{P}_{-k}\right) \leq 0$. Designaremos con la expresión $B\left(G_{n}\right)$ al conjunto de dichos equilibrios, donde $n$ es el número de empresas que pertenecen a $N$.

En una situación de equilibrio de Bertrand, entonces, encontraremos siempre un grupo de empresas (las que pertenecen al conjunto $N$ ) que estarán activas (es decir, vendiendo cantidades positivas del bien bajo análisis) y obteniendo beneficios no negativos, y otro grupo de empresas (las que no pertenecen al conjunto $N$ ) que estarán inactivas y obteniendo beneficios nulos. Adicionalmente, y en virtud de la definición de demanda individual que utilizamos, todas las empresas activas deberán estar cobrando el mismo precio $P_{B}$ (al que denominaremos "precio de equilibrio de Bertrand"). La misma definición nos asegura también que, en todo equilibrio de Bertrand, las empresas inactivas estarán cobrando un precio $P_{k}$, mayor que $P_{B}$. Este último hecho será justamente la causa por la cual dichas empresas permanecerán inactivas en equilibrio.

El lector podrá notar también que el modelo utilizado en el presente trabajo es en algún sentido similar al que aparece en trabajos referidos al tema del número de empresas en equilibrio bajo competencia monopolística. La gran diferencia entre aquellos modelos y éste, sin embargo, está en que cuando hay competencia monopolística hay también diferenciación de productos, y por lo tanto la demanda individual de cada empresa es sustancialmente diferente a la que suponemos en el presente artículo. Dicha diferenciación de productos es la que hace, por ejemplo, que bajo competencia monopolística sea posible que existan varias empresas activas cobrando precios diferentes (por productos levemente diferenciados), cosa que no puede ocurrir en un modelo de competencia de Bertrand con productos homogéneos como el descrito en el presente trabajo.

\section{Resultados}

\subsection{Resultados preliminares}

Antes de proceder a demostrar los principales resultados de este trabajo, enunciaremos y probaremos una serie de lemas que nos resultarán útiles en dicha tarea, y que son los siguientes:

Lema 1: Si Pn $\in B\left(G_{n}\right)$, entonces $P_{B} \geq P_{L}(n)$.

Prueba: De la definición de equilibrio de Bertrand sabemos que, para todo $i \in N, P_{i}=P_{B}$. Si $P_{L}(n)=\min \{P: A C(D(P) / n)=P\}$, entonces no existe ningún $P_{B}<P_{L}(n)$ para el cual se dé que $P_{B} \geq A C\left(D\left(P_{B}\right) / n\right)$. Por ende no puede haber ningún $P_{B}<P_{L}(n)$ para el cual se dé que $\Pi_{i} \geq 0$, y consecuentemente no puede haber tampoco un vector de precios $\boldsymbol{P n} \in B\left(G_{n}\right)$ para el cual se dé que $P_{B}<P_{L}(n)$, q.e.d. 
Lema 2: Si Pn $\in B\left(G_{n}\right)$, entonces $P_{B} \leq P_{H}(n)$.

Prueba: $\operatorname{Si} P_{H}(n)=\max \{P: A C(D(P) / n)=P\}$, entonces no existe ningún $P_{B}>P_{H}(n)$ para el cual se dé que $P_{B} \geq A C\left(D\left(P_{B}\right) / n\right)$. Por ende no puede haber ningún $P_{B}>P_{H}(n)$ para el cual se dé que $\Pi_{i} \geq 0$, y consecuentemente no puede haber tampoco un vector de precios $\boldsymbol{P n} \in B\left(G_{n}\right)$ para el cual se dé que $P_{B}>P_{H}(n)$, q.e.d.

Lema 3: Si Pn $\in B\left(G_{n}\right)$, entonces $P_{B} \leq P_{L}(n+1)$.

Prueba: Supóngase, por el contrario, que $P_{B}>P_{L}(n+1)$. Entonces recuérdese que, por definición, $P_{L}(n+1)=\min \{P: A C[D(P) /(n+1)]=P\}$, por lo cual, si $n+1$ empresas cobran $P_{B}$, obtienen un beneficio positivo. En ese caso $\boldsymbol{P n}$ no puede ser un equilibrio en el que sólo $n$ empresas estén activas, y consecuentemente $\boldsymbol{P n} \notin B\left(G_{n}\right)$, q.e.d.

Lema 4: Si Pn $\in B\left(G_{n}\right)$, entonces $P_{B} \leq P_{L}(1)$.

Prueba: Supóngase, por el contrario, que $P_{B}>P_{L}(1)$. Entonces la k-ésima empresa podría fijar un precio $P_{\tilde{\imath}}=P_{B}-P_{\tilde{\imath}}>P_{L}(1)$, y obtener $\Pi_{k}=P_{\tilde{\imath}} \cdot D$ $\left(P_{\imath}\right)-V C\left[D\left(P_{\tilde{l}}\right)\right]-F>0$. En ese caso $\boldsymbol{P} n$ no puede ser un equilibrio, y consecuentemente $\boldsymbol{P n} \notin B\left(G_{n}\right)$, q.e.d.

Combinando los lemas 1, 2, 3 y 4, y la definición de equilibrio de Bertrand, es posible probar un primer resultado importante que establece condiciones necesarias y suficientes para la existencia del equilibrio. Dicho resultado es la proposición 1.

Proposición 1: Supóngase que $n \geq 2$. Entonces $B\left(G_{n}\right) \neq \varnothing$ si y solo si $P_{L}(n) \leq P_{L}(n+1)$ y $P_{L}(n) \leq P_{L}(1)$.

Prueba: Combinando los lemas 1 y 3 obtenemos que, si $B\left(G_{n}\right) \neq \varnothing$, entonces $P_{L}(n) \leq P_{L}(n+1)$. Combinando los lemas 1 y 4 obtenemos que, si $B\left(G_{n}\right) \neq \emptyset$, entonces $P_{L}(n) \leq P_{L}(1)$. Por lo tanto, si $B\left(G_{n}\right)$ no está vacío, entonces $P_{L}(n) \leq P_{L}(n+1)$ y $P_{L}(n) \leq P_{L}(1)$.

Recuérdese ahora que, si $P_{L}(n) \leq P_{L}(n+1)$ y $P_{L}(n) \leq P_{L}(1)$, entonces existe un conjunto no vacío de precios $\left[P_{L}(n) ; P_{L}(n+1)\right] \cap\left[P_{L}(n) ; P_{L}(1)\right] \cap\left[P_{L}(n)\right.$; $\left.P_{H}(n)\right]$. Si $P_{B}$ pertenece a dicho conjunto y $n \geq 2$, será un precio de equilibrio de Bertrand, porque todas las empresas activas preferirán cobrar $P_{B}$ y permanecer activas en vez de cobrar $P_{i}>P_{B}$ y volverse inactivas, todas las empresas inactivas preferirán cobrar $P_{k}>P_{B}$ en vez de $P_{k}=P_{B}$ y volverse activas, y todas las empresas (activas e inactivas) preferirán quedarse como están en vez de cobrar $P_{i}<P_{B}$ y convertirse en los únicos oferentes de todo el mercado. Por lo tanto, si $n \geq 2$ y $P_{B} \in\left[P_{L}(n) ; P_{L}(n+1)\right] \cap\left[P_{L}(n) ; P_{L}(1)\right] \cap\left[P_{L}(n) ; P_{H}(n)\right]$, entonces $\boldsymbol{P n} \in B\left(G_{n}\right)$, y $B\left(G_{n}\right)$ no está vacío.

Combinando los dos resultados para el caso donde $n \geq 2$, vemos que $B\left(G_{n}\right) \neq \varnothing$ si y sólo si $P_{L}(n) \leq P_{L}(n+1)$ y $P_{L}(n) \leq P_{L}(1)$, q.e.d. 
La idea básica detrás del resultado expresado en la proposición 1 tiene que ver con cierta relación que tiene que haber entre los precios de autofinanciamiento para que el equilibrio de Bertrand pueda existir con determinado número de empresas activas. Lo que surge de ella es que, si el número de empresas activas en equilibrio es igual a $n$, entonces el precio de equilibrio $P_{B}$ tiene forzosamente que ser mayor o igual que el precio de autofinanciamiento correspondiente a una situación con $n$ empresas activas $\left(P_{L}(n)\right)$ pero, al mismo tiempo, tiene que ser menor o igual que el precio de autofinanciamiento con $n+1$ empresas activas $\left(P_{L}(n+1)\right)$ y que el precio de autofinanciamiento con una sola empresa activa $\left(P_{L}(1)\right)$. Si eso no ocurre, entonces no puede haber un equilibrio de Bertrand con $n$ empresas activas. Si $P_{B}$ es menor que $P_{L}(n)$, las empresas activas tienen beneficios negativos y entonces van a preferir volverse inactivas. $\mathrm{Si}$, en cambio, $P_{B}$ es mayor que $P_{L}(n+1)$, entonces alguna empresa inactiva tendrá incentivo a volverse activa (y el número de empresas en equilibrio no será más $n$ sino $n+1$ ). Y finalmente, si $P_{B}$ es mayor que $P_{L}(1)$, entonces alguna empresa inactiva tendrá incentivo a bajar su precio por debajo de $P_{B}$ y monopolizar el mercado (con lo cual el número de empresas activas pasará a ser uno en vez de $n$ ).

\subsection{Número óptimo de empresas y equilibrios de Bertrand}

Estamos ahora en posición de probar el resultado principal de este trabajo, léase, la relación entre el número óptimo de empresas en un mercado y la existencia del equilibrio de Bertrand para dicho número de empresas. Dicho resultado es la proposición 2 .

Proposición 2: Si $n^{*} \geq 2$ es el número óptimo de empresas en el mercado, entonces $B\left(G_{n *}\right) \neq \varnothing$.

Prueba: Recuérdese que, si $n^{*}$ es el número óptimo de empresas en el mercado, $P_{L}\left(n^{*}\right) \leq P_{L}(m)$, para todo $m \neq n^{*}$. Entonces, en particular, se da que $P_{L}\left(n^{*}\right) \leq P_{L}(n+1)$ y $P_{L}\left(n^{*}\right) \leq P_{L}(1)$. Si eso es así y $n^{*} \geq 2$, entonces por la proposición 1 sabemos que $B\left(G_{n^{*}}\right)$ no está vacío, q.e.d.

Habiendo probado la existencia del equilibrio de Bertrand cuando el número de empresas activas es el óptimo (y $\left.n^{*} \geq 2\right)$, probaremos ahora un resultado adicional, relacionado con la inexistencia del equilibrio de Bertrand cuando el número de empresas activas es menor que el número óptimo de empresas en el mercado. Para ello es conveniente enunciar primero el siguiente lema:

Lema 5: Si $n^{*}$ es el número óptimo de empresas en el mercado y $m<n^{*}$, entonces $P_{L}(m)>P_{L}(m+1)$.

Prueba: Si $n^{*}$ es el número óptimo de empresas en el mercado y $m$ no es el número óptimo, entonces $P_{L}\left(n^{*}\right)<P_{L}(m)$. Si $m=n^{*}-1$, por lo tanto, $P_{L}(m)>P_{L}(m+1)=P_{L}\left(n^{*}\right)$. Pero si $m<n^{*}-1$, entonces $Q_{i}=D\left[P_{L}(m)\right] / m$ tiene que ser una cantidad para la cual $A C\left(Q_{i}\right)$ es creciente. Por ende, como $m<m+1$, debe darse que $A C\left[D\left(P_{L}(m)\right) / m\right]>A C\left[D\left(P_{L}(m+1)\right) /(m+1)\right]$, y consecuentemente $P_{L}(m)>P_{L}(m+1)$. Combinando los dos resultados se obtiene que, si $m<n^{*}$, entonces $P_{L}(m)>P_{L}(m+1)$, q.e.d. 
Si ahora combinamos los resultados de los lemas 1,3 y 5 , resulta posible probar la siguiente proposición:

Proposición 3: Si $n *$ es el número óptimo de empresas en el mercado y $m<n *$, entonces $B\left(G_{m}\right)=\varnothing$.

Prueba: De aplicar el lema 5 sabemos que, si $n *$ es el número óptimo de empresas en el mercado y $m<n^{*}$, entonces $P_{L}(m)>P_{L}(m+1)$. Pero de aplicar los lemas 1 y 3 sabemos que, si $B\left(G_{m}\right) \neq \varnothing$, entonces $P_{L}(m) \leq P_{L}(m+1)$. Por ende, si $P_{L}(m)>P_{L}(m+1)$, entonces $B\left(G_{m}\right)$ está vacío, q.e.d.

La inexistencia del equilibrio de Bertrand con menos empresas activas que el óptimo, sin embargo, no descarta la posibilidad de encontrar equilibrios de Bertrand con más empresas activas que el número óptimo. Esto ocurre si el número de empresas activas $(m)$ es mayor que el número óptimo de empresas y, al mismo tiempo, se da que $P_{L}(m) \leq P_{L}(1)$.

Proposición 4: Supóngase que $n^{*} \geq 2$ es el número óptimo de empresas en el mercado, y que $m>n^{*}$. Entonces, si $P_{L}(m) \leq P_{L}(1), B\left(G_{m}\right) \neq \emptyset$.

Prueba: Si $n^{*}$ es el número óptimo de empresas en el mercado y $m>n^{*}$, entonces $Q_{i}=D\left[P_{L}(m)\right] / m$ tiene que ser una cantidad para la cual $A C\left(Q_{i}\right)$ es decreciente. Por ende, como $m+1>m$, debe darse que $A C\left[D\left(P_{L}(m+1)\right)\right]$ $(m+1)]>A C\left[D\left(P_{L}(m+1)\right) / m\right]$, y consecuentemente $P_{L}(m+1)>P_{L}(m)$. Si, adicionalmente, $P_{L}(m) \leq P_{L}(1)$, entonces la proposición 1 nos dice que $B\left(G_{m}\right)$ no está vacío, q.e.d.

Las proposiciones 3 y 4 ilustran la relación que tiene en nuestro modelo el concepto de equilibrio de Bertrand con el concepto de rendimientos variables a escala mencionado en la introducción. En efecto, como la función de costos que hemos supuesto para nuestras empresas tiene un componente fijo y otro componente variable convexo, entonces la misma genera una función de costo variable $\left(A C\left(Q_{i}\right)\right)$ que es decreciente hasta cierta cantidad y que luego se vuelve creciente. Esto hace que los rendimientos a escala implícitos en dicha función de costos sean variables, siendo primero crecientes (para el tramo en el cual $A C\left(Q_{i}\right)$ es decreciente) y luego crecientes (para el tramo en el cual $A C\left(Q_{i}\right)$ es creciente).

Ahora bien, de la definición de número óptimo de empresas $\left(n^{*}\right)$ surge que dicho número no es otro que aquél para el cual se da que $Q_{i}=D\left[P_{L}(n *)\right] / n *$ es la cantidad más cercana posible al punto en el cual los rendimientos dejan de ser crecientes y pasan a ser decrecientes. Esto implica que, si $m>n *$, entonces $Q_{i}=D\left[P_{L}(m)\right] / m$ tiene que ser una cantidad que se encuentra en el tramo decreciente de $A C\left(Q_{i}\right)$ y que, si $m<n^{*}$, entonces $Q_{i}=D\left[P_{L}(m)\right] / m$ tiene que ser una cantidad que se encuentra en el tramo creciente de $A C\left(Q_{i}\right)$.

Lo expresado en el párrafo anterior es precisamente lo que hace que en ciertos casos sea posible que existan equilibrios de Bertrand con un número de empresas mayor que $n^{*}$ pero que no puedan existir equilibrios con un número de empresas menor que $n^{*}$. En efecto, si $m>n^{*}$, la forma decreciente de $A C\left(Q_{i}\right)$ en el tramo relevante hará que, si existe, $P_{L}(m+1)$ sea mayor que $P_{L}(m)$. Inversamente, si $m<n^{*}$, entonces la forma creciente de $A C\left(Q_{i}\right)$ en el tramo relevante será la que haga que $P_{L}(m+1)$ sea menor que $P_{L}(m)$. En este último caso, por lo tanto, no 
será posible sostener un equilibrio de Bertrand con $m$ empresas, ya que siempre habrá un incentivo para que alguna empresa inactiva quiera entrar al mercado y el número de empresas activas pase a ser $m+1$ (en vez de $m$ ).

\subsection{El caso de una sola empresa activa}

Quizás el lector haya notado que, en las proposiciones 1, 2 y 4, nos hemos restringido a situaciones en las que el número de empresas activas en el mercado es mayor o igual que dos. Esto se debe a que, bajo nuestra definición del espacio de estrategias de las empresas y en virtud de la regla de reparto elegida para definir las demandas individuales, $B\left(G_{1}\right)$ es siempre un conjunto vacío.

Para el caso en el que el número óptimo de empresas activas es $n^{*} \geq 2$, el carácter vacío del conjunto $B\left(G_{l}\right)$ puede probarse aplicando de manera directa la proposición 3 al caso particular en el que $m=1$. Cuando el número óptimo de empresas activas es uno, en cambio, $B\left(G_{m}\right)$ está vacío para todo $m \geq 1$, incluyendo a $m=1$ como un caso especial. Esto puede probarse del siguiente modo:

Proposición 5: Si el número óptimo de empresas en el mercado es uno, entonces $B\left(G_{m}\right)=\varnothing$ para todo $m \geq 1$.

Prueba: Combinando los lemas 1 y 4 , sabemos que si $B\left(G_{m}\right) \neq \varnothing$, entonces $P_{L}(m) \leq P_{L}(1)$. Pero si el número óptimo de empresas activas es uno, entonces $P_{L}(m)>P_{L}(1)$ para todo $m \geq 2$, y por ende $B\left(G_{m}\right)=\emptyset$ para todo $m \geq 2$.

$\mathrm{Si}$, en cambio, el número óptimo de empresas activas es uno y $m=1$, la combinación de los lemas 1 y 4 implica que $P_{L}(1) \leq P_{B} \leq P_{L}(1)$, con lo cual el único posible precio de equilibrio de Bertrand sería $P_{B}=P_{L}(1)$. Pero si la única empresa activa está cobrando $P_{B}=P_{L}(1)$, entonces todas las empresas inactivas tienen que estar cobrando $P_{k}>P_{L}(1)$. Debe por lo tanto existir algún $P_{\tilde{\imath}}=P_{k}-\varepsilon>P_{L}(1)$ para el cual la empresa activa puede obtener un beneficio positivo, y por lo tanto $P_{L}(1)$ no puede ser un precio de equilibrio y $B\left(G_{1}\right)=\varnothing$.

Por ende, si el número óptimo de empresas en el mercado es uno, entonces $B\left(G_{m}\right)$ está vacío para todo $m \geq 1$, q.e.d.

Nótese que decir que el equilibrio de Bertrand no existe cuando el número óptimo de empresas en el mercado es uno es equivalente a decir que $B\left(G_{m}\right)$ está vacío para cualquier $m \geq 1$ si el mercado es un monopolio natural. Si el mercado no es un monopolio natural, en cambio, sabemos por la proposición 2 que siempre podremos encontrar una asignación de equilibrio de Bertrand, al menos para el caso en el cual el número de empresas activas sea igual al número óptimo de empresas en el mercado.

La inexistencia del equilibrio de Bertrand en una situación como esta, sin embargo, depende de manera esencial de la regla de reparto de la demanda que estamos utilizando en este trabajo para los casos en los cuales dos o más empresas fijan el mismo precio (que es la regla de reparto igualitario). Si cambiáramos dicha regla (como hace Hoernig, 2007), podríamos hallar un equilibrio de Bertrand con una empresa activa que abastece todo el mercado y algunas otras empresas inactivas que cobraran el mismo precio $P_{B}$ que la empresa activa. También resultaría posible hallar equilibrios con una sola empresa activa si definiéramos el espacio de estrategias de modo de que el precio sea una variable discreta y no continua (como ocurre en Chaudhuri, 1998, y en Chowdhury, 2002). 
Si el precio se transforma en una variable discreta se abre también una vía alternativa para analizar posibles equilibrios de Bertrand en estrategias mixtas (en vez de equilibrios en estrategias puras, como son todos los que analizamos en el presente trabajo). Dichos equilibrios también han sido explorados por la literatura en modelos en los cuales la variable estratégica de las empresas no es el precio sino la decisión de entrar o no entrar al mercado, y en los cuales es común que el equilibrio quede determinado como un conjunto de probabilidades de que cada una de las empresas participantes entre o no al mercado ${ }^{3}$.

\section{EJEMPLO NUMÉRICO}

Los resultados de la sección anterior nos muestran que, en este tipo de mercados donde hay libre entrada de empresas idénticas con rendimientos variables a escala, el equilibrio de Bertrand existe si el número óptimo de empresas es mayor o igual que dos y el número de empresas activas es el óptimo. También nos dicen que dicho equilibrio no existe si el número óptimo de empresas es igual a uno, tampoco si el número de empresas activas es menor que el óptimo, pero que puede existir si el número óptimo de empresas es mayor o igual que dos y el número de empresas activas es mayor que el óptimo. Esto es lo que nos mostrará el siguiente ejemplo numérico.

Considérese una situación en la cual $C\left(Q_{i}\right)=0,5 \cdot Q_{i}^{2}+3$ cuando $Q_{i}>0 \mathrm{y}$ $C(0)=0$, y supóngase que la función de demanda agregada es $Q=12-2 \cdot P$. Entonces la correspondiente función de costo medio con $n$ empresas activas es $A C(Q / n)=0,5 \cdot(Q / n)+3 \cdot n / Q$. Con estas funciones, los precios de autofinanciamiento para los distintos números posibles de empresas activas son $P_{L}(1)=3,2753$, $P_{L}(2)=2,5858, P_{L}(3)=2,451, P_{L}(4)=2,6202$ y $P_{L}(5)=3.5$. Cuando $n \geq 6$, $P_{L}(n)$ no existe, porque la función de demanda agregada no se cruza con $A C(Q / n)$. Observando los diferentes valores de $P_{L}(n)$, podemos hallar que el número óptimo de empresas activas en este mercado es tres, ya que $P_{L}(3)=2,451$ es el mínimo valor posible para $P_{L}(n)=A C(D(P) / n)$. La cantidad demandada en una situación en la cual $P=P_{L}(3)$, por su parte, es $Q=7,098$.

Que tres es el número óptimo de empresas activas cuando $Q=7,098$ puede deducirse también de inspeccionar las funciones de costo medio para distintos números de empresas activas, y encontrar cuál es el mínimo $A C(Q / n)$ para cada posible valor de $Q$. Haciendo eso encontramos que el número óptimo de empresas activas es uno para $Q \leq 3,4641$, dos para $3,4641 \leq Q \leq 6$, tres para $6 \leq Q \leq 8,4853$, y cuatro para $8,4853 \leq Q \leq 10,9545$. Como $Q=7,098$ pertenece al intervalo [6; 8,4853], el número óptimo de empresas es tres.

$\mathrm{Si}$ hay tres empresas activas en este mercado, el conjunto de posibles precios de equilibrio de Bertrand está dado por el intervalo $\left[P_{L}(3)=2,451\right.$; $\left.P_{L}(4)=2,6202\right]$, que es el rango para el cual tres empresas activas obtienen beneficios positivos pero una cuarta empresa obtendría beneficios negativos si se volviera activa. Ningún equilibrio de Bertrand existe para $n=1$ ó $n=2$ ya que, si $P=P_{L}(1)=3,2753$, una segunda empresa encontraría beneficioso entrar al mercado y, si $P=P_{L}(2)=2,5858$, una tercera empresa encontraría beneficioso hacerlo. Si $n=4$, en cambio, existe un conjunto no vacío de posi-

$3 \quad \mathrm{Al}$ respecto véase, por ejemplo, Vettas (2000). 
bles precios de equilibrio de Bertrand, dado por el intervalo $\left[P_{L}(4)=2,6202\right.$; $\left.P_{L}(1)=3,2753\right]$. En ese rango de precios, las cuatro empresas activas obtienen beneficios no negativos, las empresas inactivas no encuentran beneficioso entrar al mercado (ni como un quinto participante en el oligopolio existente ni como un monopolista), y ninguna empresa activa tiene tampoco incentivos para bajar su precio y volverse monopolista.

Si $n \geq 5$, por último, el equilibrio de Bertrand no existe en este ejemplo numérico. Esto se debe a que, cuando $P=P_{L}(5)=3,5$, cualquier empresa prefiere bajar su precio y convertirse en monopolista (ya que $P_{L}(1)<3,5$ ). Y como mencionamos antes, $P_{L}(n)$ no existe cuando $n \geq 6$, y por lo tanto es imposible hallar una asignación en la cual seis o más empresas estén activas y todas ellas obtengan beneficios no negativos.

El Gráfico 1 es una representación de nuestro ejemplo numérico. En él hemos dibujado la demanda agregada $D(P)$, y las funciones de costo medio para distintos números de empresas activas. Vemos que el número óptimo de empresas en este mercado es $n=3$, ya que el menor precio al cual $D(P)$ cruza una función de costo medio $\left(P_{L}(3)\right)$ corresponde a un punto ubicado en $A C(Q / 3)$. Ese precio es uno de los posibles precios de equilibrio de Bertrand, junto con todos los precios en el intervalo $\left[P_{L}(3), P_{L}(4)\right]$, cuando hay tres empresas activas, y todos los precios en el intervalo $\left[P_{L}(4), P_{L}(1)\right]$, cuando el número de empresas activas es cuatro.

Otra manera de representar el mismo ejemplo numérico es la que aparece en el Gráfico 2, en el cual hemos dibujado una única función de costo medio $(A C(Q i))$ y seis funciones distintas de demanda individual $(D(P), D(P) / 2, D(P) / 3$, $D(P) / 4, D(P) / 5$ y $D(P) / 6)$, correspondientes a los casos en los cuales hay una, dos, tres, cuatro, cinco y seis empresas activas en el mercado. Aquí también puede observarse que el mínimo precio de autofinanciamiento es $P_{L}(3)$, y que, mientras $D(P)$ y $D(P) / 2$ cruzan a $A C(Q i)$ en un tramo en el cual esta última

\section{GRÁFICO 1}

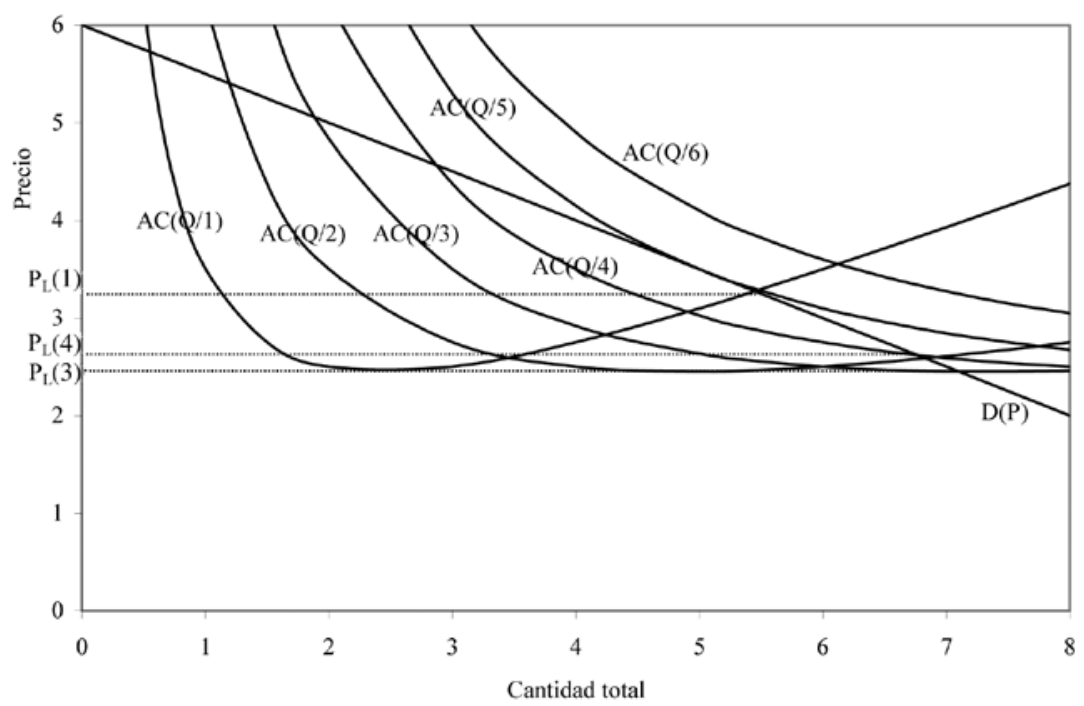




\section{GRÁFICO 2}

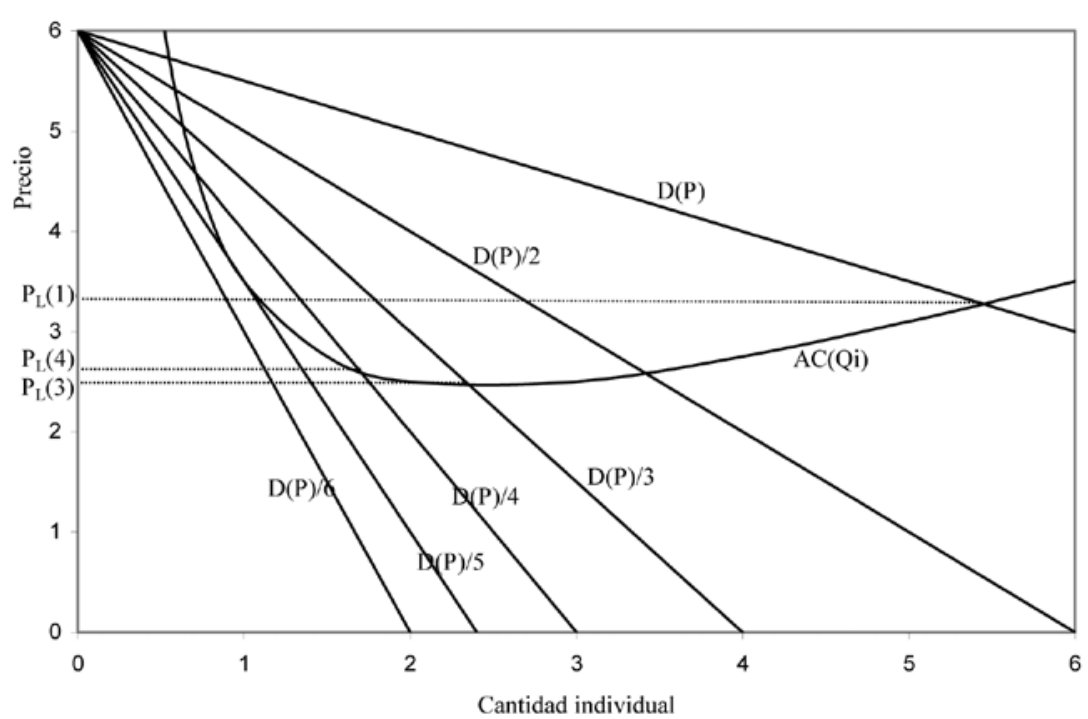

función es creciente, $D(P) / 4$ y $D(P) / 5$ lo hacen en un tramo en el cual $A C(Q i)$ es decreciente. Como, además, $P_{L}(1)$ es mayor que $P_{L}(4)$ y que $P_{L}(3)$, se ve entonces que los intervalos $\left[P_{L}(3), P_{L}(4)\right]$ y $\left[P_{L}(4), P_{L}(1)\right]$ son capaces de sostener precios de equilibrio de Bertrand cuando el mercado tiene tres y cuatro empresas activas, respectivamente.

Un punto interesante que puede verse en nuestro ejemplo numérico es que el número de empresas activas en el mercado típicamente aumenta cuando se produce un crecimiento en la demanda. Supongamos, por ejemplo, que la demanda agregada se incrementa de manera proporcional en un $40 \%$ y pasa a ser $Q=16,8-2.8 \cdot P$. Si la función de costo total de las empresas no se modifica, esto implica que ahora los precios de autofinanciamiento para los distintos números posibles de empresas activas son $P_{L}(1)=3,6936, P_{L}(2)=2,8734$, $P_{L}(3)=2,543, P_{L}(4)=2,4497, P_{L}(5)=2,5126, P_{L}(6)=2,7283$ y $P_{L}(7)=3,5$. Cuando $n \geq 8$, por su parte, $P_{L}(n)$ no existe. El número óptimo de empresas activas en este mercado es ahora cuatro, ya que $P_{L}(4)=2,4497$ es el mínimo valor posible para $P_{L}(n)=\min \{P: A C(D(P) / n)=P\}$.

Aplicando los resultados de nuestro modelo a esta nueva situación, vemos que los posibles precios de equilibrio de Bertrand son los contenidos en los intervalos $\left[P_{L}(4)=2,4497 ; P_{L}(5)=2,5126\right]$, cuando hay cuatro empresas activas; $\left[P_{L}(5)=2,5126 ; P_{L}(6)=2,7283\right]$, cuando hay cinco empresas activas; $\mathrm{y}$ $\left[P_{L}(6)=2,7283 ; P_{L}(7)=3.5\right]$, cuando hay seis empresas activas. Existe tambiến un equilibrio con siete empresas activas en cual los oferentes cobran $P_{B}=P_{L}(7)=P_{H}(7)=3,5$, ya que en esa situación todos obtienen beneficios nulos, pero el precio es menor que el precio de autofinanciamiento para un monopolista $\left(P_{L}(1)=3,6936\right)$. No hay, en cambio, equilibrios de Bertrand con ocho o más empresas activas, debido a que en tales casos la demanda individual 
no se cruza en ningún punto con la función de costo medio. Tampoco hay equilibrios con tres o menos empresas activas, ya que en esos casos existe siempre un incentivo para la entrada de una nueva empresa.

Lo expuesto puede verse en el Gráfico 3, que es semejante al Gráfico 2 pero se refiere a esta nueva situación en la cual la demanda agregada ha crecido un $40 \%$. Nótese que ahora $A C(Q i)$ es creciente en el tramo en el cual cruza a $D(P), D(P) / 2$ y $D(P) / 3$, en tanto que es decreciente en el tramo en el cual cruza a $D(P) / 5, D(P) / 6$ y $D(P) / 7$.

De la comparación de los dos ejemplos numéricos expuestos, puede extraerse una conclusión preliminar respecto del número de empresas de equilibrio en diferentes casos de cambios en la demanda. Supongamos, por ejemplo, que estamos en una situación en la que la demanda agregada es la que postulamos al principio $(Q=12-2 \cdot P)$ y que, por lo tanto, el número de empresas en el mercado es tres o cuatro. Si en cierto momento la demanda agregada se incrementa en un $40 \%$ y pasa a ser la postulada en nuestro segundo ejemplo numérico $(Q=16,8-2,8 \cdot P)$, entonces ahora existirá un incentivo para que ingrese al mercado una cuarta empresa activa (si antes no la había), o bien para que las cuatro empresas preexistentes reacomoden sus precios a las nuevas condiciones, y el equilibrio pasará a ser uno con el número óptimo de empresas activas (en este caso, cuatro).

$\mathrm{Si}$, en cambio, nos hallamos inicialmente en una situación en la cual la demanda agregada es $Q=16,8-2,8 \cdot P$, ya hay cuatro empresas en el mercado, y lo que se produce es una reducción de dicha demanda; entonces lo más probable es que las cuatro empresas permanezcan (por más que el número óptimo de empresas sea ahora tres). Esto se debe a que, cuando $Q=12-2 \cdot P$, el equilibrio de Bertrand con cuatro empresas también existe, y es por lo tanto probable que

\section{GRÁFICO 3}

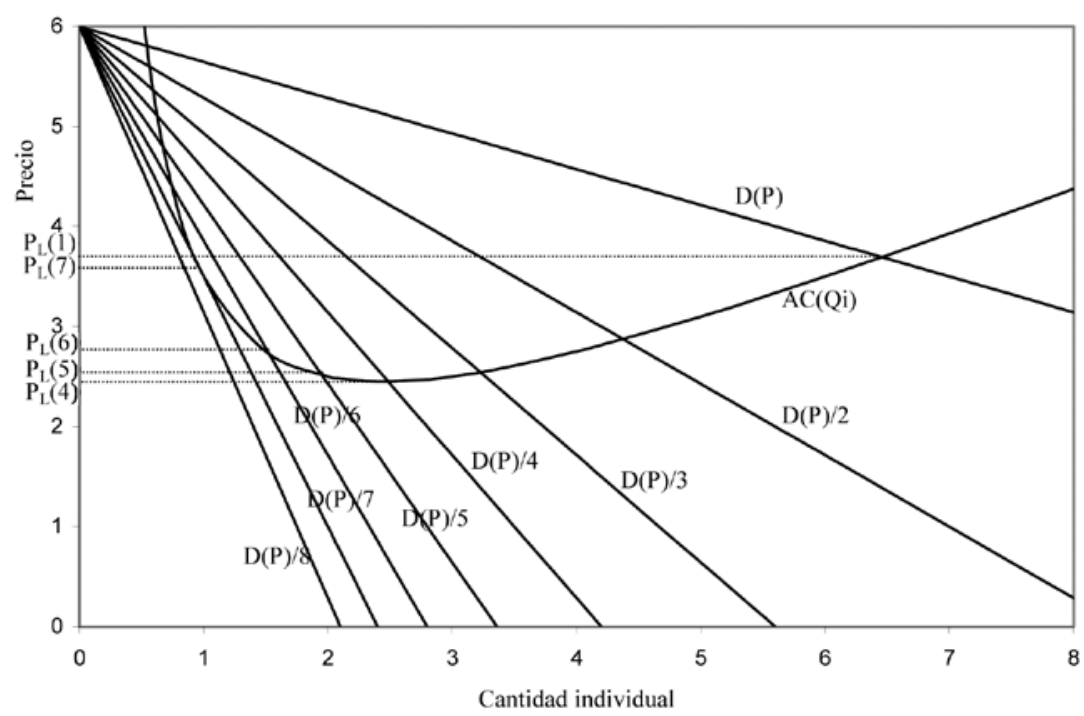




\section{GRÁFICO 4}

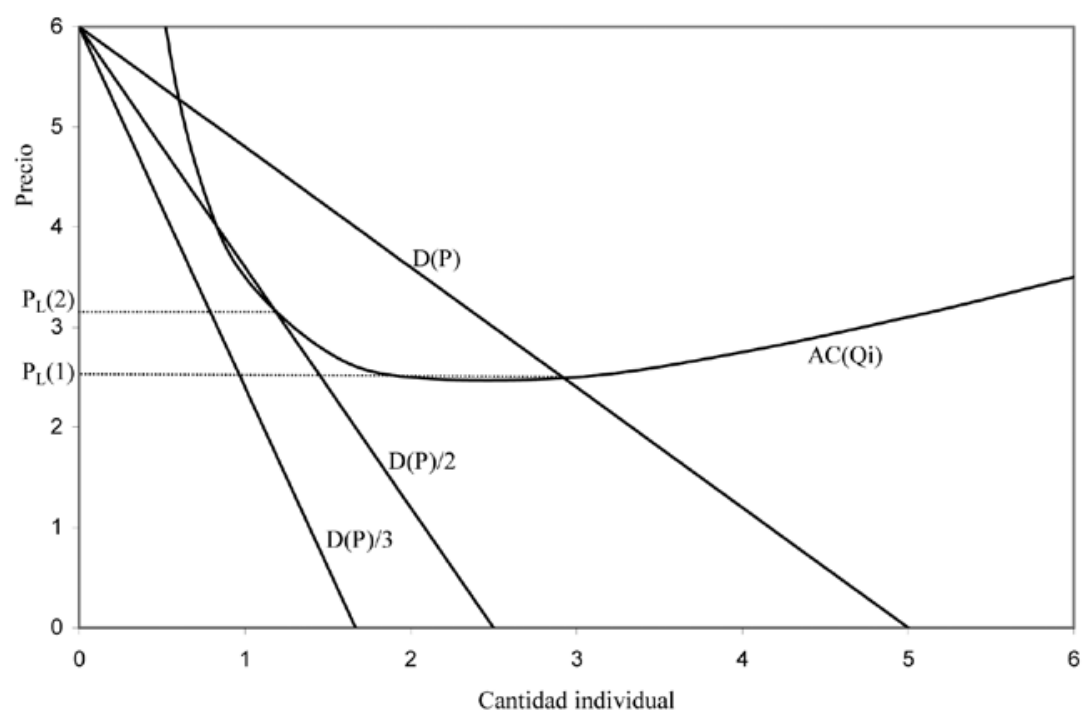

el ajuste que se produzca sea tal que ninguna de dichas empresas abandone el mercado y el precio se ubique en un rango entre $P_{L}(4)$ y $P_{L}(1)$. Esto nos indica que la competencia de Bertrand puede no ser capaz de generar el número óptimo de empresas en el mercado, especialmente si estamos en una situación en la cual la demanda agregada está disminuyendo.

Una última versión de nuestro ejemplo numérico puede ser un caso en el cual el número óptimo de empresas en el mercado es igual a uno (monopolio natural). Supongamos, por ejemplo, que la demanda agregada es $Q=5-0,833 \cdot P$, y que por lo tanto $P_{L}(1)=2,4883, P_{L}(2)=3,064$, y $P_{L}(n)$ no existe para $n \geq 3$. En un caso como este, representado en el Gráfico 4 , el único equilibrio posible sería que hubiera una sola empresa activa cobrando $P_{B}=P_{L}(1)=2,4883$, pero en dicha situación las empresas inactivas deberían estar cobrando precios superiores a $P_{L}(1)$. La empresa activa, por lo tanto, podría hallar siempre un precio $P_{\tilde{\imath}}=P_{k}-\varepsilon>P_{L}(1)$, que le permitiera obtener un beneficio mayor. Se da así el caso en el cual el equilibrio de Bertrand, al menos tal como lo hemos definido en el presente trabajo, no existe para ningún $n \geq 1$.

\section{Comentarios finales}

La principal conclusión de este trabajo es que el número óptimo de empresas, en el mercado de un producto homogéneo con rendimientos variables a escala y libre entrada de empresas idénticas, puede sostenerse como el resultado de un proceso de competencia de Bertrand cuando dicho número óptimo de empresas es mayor o igual que dos (proposición 2). El trabajo nos muestra también que ese proceso puede generar resultados en los que el número de empresas activas es 
mayor que el óptimo (proposición 4), pero no resultados en los que tal número es menor que el óptimo (proposición 3 ).

Si el número óptimo de empresas es mayor o igual que dos, lo que típicamente aparece es una situación con múltiples equilibrios de Bertrand, que sostienen todo un rango de precios (proposición 1). En todos esos equilibrios las empresas activas eligen el mismo precio, y el límite inferior del intervalo es el precio de autofinanciamiento de la industria $\left(P_{L}(n)\right)$. Este resultado es esencialmente el mismo encontrado por Dastidar (1995) para equilibrios de Bertrand en mercados con rendimientos decrecientes a escala sin libre entrada. La principal diferencia con el resultado de Dastidar es que, en nuestro modelo, el límite superior del conjunto de precios de equilibrio está dado por el precio de autofinanciamiento de la industria correspondiente a $n+1$ empresas activas $\left(P_{L}(n+1)\right)$ o bien por el precio de autofinanciamiento de la industria correspondiente a una sola empresa activa $\left(P_{L}(1)\right)$. Esto se debe a que la existencia de empresas inactivas (competidores potenciales) obliga a las empresas activas a fijar precios que disuadan a las empresas inactivas de entrar al mercado, sea como nuevos participantes en el oligopolio existente o como monopolistas ${ }^{4}$.

El resultado referido a la posibilidad de equilibrios de Bertrand con distintos números de empresas activas en el mercado (proposición 4) también es consistente con el que hallaron D'Aspremont, Ferreira y Gérard-Varet (2000), quienes mostraron que el número de empresas activas en un equilibrio con libre entrada podía quedar indeterminado bajo distintas especificaciones del mercado. Usando un modelo parecido al nuestro, Novshek y Chowdhury (2003) probaron también que el rango de posibles precios de equilibrio de Bertrand bajo rendimientos variables a escala es típicamente creciente cuando la demanda aumenta. Esto se debe a que pueden existir equilibrios de Bertrand con más empresas activas que el óptimo, y las posibilidades son crecientes cuando el número óptimo de empresas se incrementa.

Por el contrario, cuando el número óptimo de empresas en el mercado es uno (es decir, cuando el mercado es un monopolio natural), la implicancia de nuestro modelo es que el equilibrio de Bertrand no existe (proposición 5). Esto es consistente con lo hallado por nosotros en un trabajo anterior (Saporiti y Coloma, 2010), en el cual, para un caso sin libre entrada, mostramos que una condición necesaria y suficiente para la existencia del equilibrio de Bertrand es que la función de costos de las empresas no sea subaditiva para $Q_{i}=D\left(P_{L}(n)\right)$. Para restaurar la existencia del equilibrio de Bertrand en una situación como ésa, es necesario cambiar la regla de reparto de la demanda para casos en los que dos o más empresas fijan el mismo precio, o bien definir el espacio de estrategias de modo de que el precio sea una variable discreta y no continua. Otro caso en el cual el equilibrio puede existir cuando el número óptimo de empresas en el mercado es uno es el llamado "equilibrio de Demsetz" (Yano, 2006), pero esto ocurre en el contexto de un modelo de Bertrand-Edgeworth y no en un modelo de competencia de Bertrand como el que utilizamos nosotros 5 .

4 Este requerimiento es similar al que aparece en Ferreira y Dufourt (2007), quienes lo denominan "condición de sostenibilidad" (sustainability condition).

5 Para una explicación de la diferencia entre la competencia de Bertrand y la de BertrandEdgeworth, véase Vives (1999), capítulo 5. 
Adicionalmente, puede decirse que nuestro modelo también tiene una implicancia relacionada con la eficiencia de los equilibrios bajo distintos cambios en la demanda. La competencia de Bertrand con libre entrada puede así ser eficiente para generar la entrada óptima de nuevas empresas, pero no para inducir la salida óptima de las empresas existentes. Esto es porque los incrementos en la demanda incentivan la entrada de nuevas empresas, pero una vez que el mercado está en equilibrio y el precio se ubica entre $P_{L}\left(n^{*}\right)$ y $P_{L}\left(n^{*}+1\right)$ no existen ya incentivos adicionales para que se produzca una entrada excesiva. Por el contrario, si la demanda está decreciendo, deberíamos esperar que el número de empresas de equilibrio fuera mayor que el óptimo (o sea, $m>n^{*}$ ), tal como se ve en el ejemplo numérico de la sección 4. Esto se debe a que, si $P_{L}(m)$ existe y el número actual de empresas activas en el mercado es $m$, entonces una asignación con $m$ empresas que cobran un precio que pertenece al conjunto $\left[P_{L}(m) ; P_{L}(m+1)\right] \cap\left[P_{L}(m) ; P_{L}(1)\right] \cap\left[P_{L}(m) ; P_{H}(m)\right]$ puede sostenerse como un equilibrio de Bertrand, aun cuando el número óptimo de empresas sea menor que $m$.

Esta implicancia de nuestro modelo respecto de la eficiencia del equilibrio de Bertrand bajo diferentes circunstancias abre también la puerta para algunas posibles conclusiones relacionadas con la política económica y la regulación de mercados. La principal tiene que ver sin duda con el hecho de que, en ciertas circunstancias, podría considerarse eficiente que el gobierno (o, inclusive, que ciertos agentes económicos privados) llevara a cabo acciones destinadas a limitar la entrada a ciertos mercados, si dicha limitación tiene como consecuencia una reducción del precio de equilibrio de largo plazo. El caso más extremo de la misma tendrá lugar en casos de monopolio natural, en el cual nuestro modelo pronostica la inexistencia de equilibrio, y podría servir para justificar la necesidad de establecer una disposición legal que garantice la existencia de una única empresa proveedora y que al mismo tiempo le impida abandonar el mercado.

\section{REFERENCIAS}

Anderson, S., A. de Palma y Y. Nesterov. (1995). "Oligopolistic competition and the optimal provision of products", Econometrica 63, 1281-1301.

Brander, J. y B. Spencer. (1985). "Tacit collusion, free entry and welfare", Journal of Industrial Economics 33, 277-294.

Chaudhuri, R. (1998). "Contestability and Bertrand equilibrium: A unified approach", Keio Economic Studies 35, 61-70.

Chowdhury, P. (2002). "Limit-pricing as Bertrand equilibrium", Economic Theory 19, 811-822.

Corchon, L. e I. Fradera. (2002). "Comparative statics in Cournot free entry equilibrium", Mathematical Social Sciences 44, 155-168.

D’Aspremont, C., R. Ferreira y L.-A. Gérard-Varet. (2000). "Contestability and the indeterminacy of free-entry equilibria", Japanese Economic Review 51, 72-83.

Dastidar, K. (1995). "On the existence of pure strategy Bertrand equilibrium", Economic Theory 5, 19-32. 
Dixit, A. y J. Stiglitz. (1977). "Monopolistic competition and optimum product diversity", American Economic Review 67, 297-308.

Ferreira, R. y F. Dufourt. (2007). "Free entry equilibria with positive profits: a unified approach to quantity and price competition games", International Journal of Economic Theory 3, 75-94.

Friedman, J. y J. Thisse. (1994). "Sustainable collusion in oligopoly with free entry", European Economic Review 38, 271-283.

Hoernig, S. (2007). "Bertrand games and sharing rules", Economic Theory 31, 573-585.

Mankiw, G. y M. Whinston. (1986). "Free entry and social inefficiency", Rand Journal of Economics 17, 48-58.

Marshall, A. (1890). Principles of economics, London, Macmillan (Hay versión en castellano: Principios de economía, Madrid, Aguilar).

Novshek, W. y P. Chowdhury. (2003). "Bertrand equilibria with entry: limit results", International Journal of Industrial Organization 21, 795-808.

Saporiti, A. y G. Coloma. (2010). "Bertrand competition in markets with fixed costs", Berkeley Electronic Journal of Theoretical Economics 10, art. 27.

Sharkey, W. y D. Sibley. (1993). "A Bertrand model of pricing and entry", Economics Letters 41, 199-206.

Vettas, N. (2000). "On entry, exit and coordination with mixed strategies", European Economic Review 44, 1557-1576.

Vives, X. (1999). Oligopoly pricing, Cambridge, MIT Press (Hay versión en castellano: Oligopolio y precios, Barcelona, Antoni Bosch).

Weibull, J. (2006). "Price competition and convex costs", Documento de Trabajo SSE/EFI 622, Stockholm School of Economics.

Yano, M. (2006). "A price competition game under free entry", Economic Theory 29, 395-414. 
